\title{
Economic, Social, and Psychological Factors Associated With Health-Related Quality of Life of Chronic Hemodialysis Patients in Northern Taiwan: A Multicenter Study
}

\author{
$*$ Tze-Wah Kao, $†$ Mei-Shu Lai, *Tun-Jun Tsai, $\$$ Chyi-Feng Jan, †Wei-Chu Chie, \\ and *Wan-Yu Chen
}

\begin{abstract}
*Division of Nephrology, Department of Internal Medicine, National Taiwan University Hospital and National Taiwan University College of Medicine; †Institute of Preventive Medicine and Department of Public Health, College of Public Health, National Taiwan University; and $\$$ Department of Family Medicine, National Taiwan University Hospital and National Taiwan University College of Medicine, Taipei, Taiwan
\end{abstract}

\begin{abstract}
This study evaluated the associations between economic, social, psychological factors, and health-related quality of life of hemodialysis patients. Cross-sectional study design was used. End-stage renal disease patients who had received maintenance hemodialysis for more than 2 months at 14 centers in northern Taiwan were invited to participate. Demographic, economic, and psychosocial data of patients were collected. Depression was assessed by the Beck Depression Inventory. Health-related quality of life was measured by the Medical Outcomes Study ShortForm 36. Multivariable linear regression analyses were performed. Eight hundred sixty-one patients (373 males, mean age $59.4 \pm 13.2$ years) completed the study. Higher
\end{abstract}

monthly income was positively associated with role emotional and mental health $(P<0.05)$, and so was increased frequency of social activities with social functioning $(P<$ $0.05)$. The more worries, the stronger the inverse associations with social functioning $(P<0.05)$ and mental health $(P<0.01)$. Higher depression scores were associated with lower scores of all Short-Form 36 dimensions $(P<0.01)$. Higher monthly income and increased social activities are associated with better health-related quality of life, whereas more worries and higher depression scores are associated with worse health-related quality of life of hemodialysis patients. Key Words: Economic-Psychosocial-Depression-Quality of life-Hemodialysis.
Survival of end-stage renal disease (ESRD) patients has been largely improved nowadays because of medical progress, advanced technology, and better patient care. Accumulated data in the recent decade show that health-related quality of life (HRQOL) markedly influences dialysis outcomes (1). Attention thus needs to be focused not only on how long but also on how well ESRD patients live (2).

Compared with the general population, ESRD patients have significantly impaired HRQOL (3), whether they are treated with hemodialysis (HD) or peritoneal dialysis (4). HRQOL is an important

doi:10.1111/j.1525-1594.2008.00675.x

Received September 2007; revised February 2008

Address correspondence and reprint requests to Professor WeiChu Chie, Room 520, No. 17 Xu-Zhou Road, Taipei, 10055, Taiwan. E-mail: weichu@ntu.edu.tw effectiveness indicator of the medical care a patient receives (5). Higher scores of HRQOL are correlated with higher self-esteem and lower levels of mood disturbance and treatment stresses (6). As HRQOL of HD patients is frequently difficult to improve by medical therapy alone, the identification of psychosocial factors that play an important role in QOL becomes crucial (7). HRQOL of HD patients in Taiwan and its association with nonmedical factors have not been widely investigated. This study aimed to determine the associations between economic, social, psychological factors, and HRQOL of HD patients in Taiwan.

\section{MATERIALS AND METHODS}

\section{Patients}

ESRD patients who had received maintenance HD for more than 2 months at 14 HD centers in 
northern Taiwan were invited to participate in this cross-sectional study. Patients who could not communicate well with others because of old age, dementia, consciousness disturbance, or severe chronic disease, or those who had intercurrent events such as surgery, infection, and hospitalization 1 month prior to the study were excluded, leaving 861 patients eligible. The study was approved by the Institutional Review Board of the National Taiwan University Hospital and relevant units of each hospital, and informed consent was obtained from every patient at the study entry.

\section{Methods of data collection}

The principal investigator and a well-trained assistant visited each study hospital and explained to the physicians and HD nurses the detailed methods of data collection and the meaning of each question on the questionnaires at the start of the study. Demographic, economic, social, and psychological data were collected through self-administered questionnaires whereas clinical data were recorded by the HD nurses. Patients who had problems in reading or understanding the questionnaires were assisted by the HD nurses. During the study period, the assistant revisited all study hospitals regularly to gather completed questionnaires.

\section{Measurement of HRQOL}

HRQOL was measured by the Medical Outcomes Study 36-Item Short Form Health Survey (SF-36) (8), a 36-item questionnaire comprising eight dimensions: physical functioning (PF); role physical (RP); bodily pain (BP); general health (GH); vitality (VT); social functioning (SF); role emotional (RE); and mental health $(\mathrm{MH})$. The SF-36 scores were then transformed to a scale of $0-100$, with higher scores indicating better HRQOL, that is, a more favorable health status. This self-assessed questionnaire has been considered a consistent and powerful predictor of morbidity and mortality in dialysis patients (9). The Chinese version (Taiwan standard version 1.0) of the SF-36 was used in this study. It was translated in 1996, and has been tested reliable (10) and valid (11). It has also been applied successfully in previous studies on ESRD patients in Taiwan $(12,13)$.

\section{Collection of demographic, economic, social, psychological, and clinical data}

Demographic data of the patients such as sex, age, marital status, educational level, religious beliefs, and place of birth were recorded. Economic factors including occupation (work status), monthly income, and sources of economic support were documented.
Sources of social support when difficulties were encountered as well as frequency of joining social activities were considered as social parameters of the patients.

Psychological factors, for example, depressive affect and the most troublesome problem(s) a patient was worrying about, were recorded. Each patient was requested to complete the 21 questions of the Beck Depression Inventory (BDI) (14), a well-validated and frequently used measure of depression for patients with ESRD (15). It asks about symptoms of depression including sadness, pessimism, experience of failure, loss of joyfulness, guilty feeling, sensation of being punished, self-dislike, self-reproach, suicide ideation, crying, irritability, loss of interest, lack of decision, sense of valueless, loss of activity, change of sleep habit, easy anger, change of appetite, decreased attention, tiredness, and loss of sex interest. BDI scores of 14 to 19 represent a mild degree of depression, 20 to 28 a moderate degree, and 29 to 60 a severe degree. This study employed the Chinese version of the BDI-II published by the Chinese Behavioral Science Corporation in Taiwan.

Clinical parameters including body height, body weight, body mass index, primary cause of renal failure, comorbid diseases, hepatitis status, duration on HD, most bothering physical symptoms, use of erythropoietin, complete cell count, blood chemistry data before $\mathrm{HD}$, and dialysis adequacy $(\mathrm{Kt} / \mathrm{V})$ were collected around study entry.

\section{Statistical analysis}

Percentage, mean, and standard deviation (SD) were used to describe the distribution of all independent variables and SF-36 scores. Through univariable linear regression analyses, the associations between HRQOL and independent variables of interest including demographic, economic, social, psychologi$\mathrm{cal}$, and clinical factors were examined. Variables with $P$ values less than 0.05 in the analyses and other variables of interest were tested with force-in multivariable linear regression models. A two-sided $P$ of 0.05 was considered the cutoff value of statistical significance. Data were reported as mean \pm SD. All analyses were performed using the SAS system version 8.1 (SAS Institute, Inc., Cary, NC, USA).

\section{RESULTS}

Eight hundred and sixty-one HD patients, among them 373 males $(43.3 \%)$, completed the study. Their demographic characteristics are displayed in Table 1, and their mean age was $59.4 \pm 13.2$ years (range $15-89$ years). The majority were born in Taiwan, with 
TABLE 1. Demographic characteristics of study HD patients

\begin{tabular}{|c|c|c|}
\hline & $\begin{array}{l}\text { Number of } \\
\text { patients }\end{array}$ & $\begin{array}{c}\text { Percentage } \\
(\%)\end{array}$ \\
\hline Age & 861 & $59.4 \pm 13.2 *$ \\
\hline \multicolumn{3}{|l|}{ Sex } \\
\hline Male & 373 & 43.32 \\
\hline Female & 488 & 56.68 \\
\hline \multicolumn{3}{|l|}{ Birth place } \\
\hline Taiwan & 751 & 88.88 \\
\hline Mainland China & 87 & 10.30 \\
\hline Aborigines & 2 & 0.23 \\
\hline Others & 5 & 0.59 \\
\hline \multicolumn{3}{|l|}{ Educational level } \\
\hline Primary school or below & 416 & 48.48 \\
\hline Junior high school & 141 & 16.43 \\
\hline Senior high school or above & 297 & 34.62 \\
\hline Others & 4 & 0.47 \\
\hline \multicolumn{3}{|l|}{ Marital status } \\
\hline Married & 615 & 71.93 \\
\hline Single & 90 & 10.53 \\
\hline Widowed & 108 & 12.63 \\
\hline Divorced & 37 & 4.33 \\
\hline Others & 5 & 0.58 \\
\hline \multicolumn{3}{|l|}{ Religious status } \\
\hline Without religious belief & 169 & 19.74 \\
\hline With religious belief & 687 & 80.26 \\
\hline \multicolumn{3}{|l|}{ Types of religion ${ }^{\dagger}$} \\
\hline Buddhism & 457 & 53.39 \\
\hline Taoism & 251 & 29.32 \\
\hline Christianity & 21 & 2.45 \\
\hline Catholics & 10 & 1.17 \\
\hline Islam & 0 & 0.00 \\
\hline Others & 6 & 0.70 \\
\hline
\end{tabular}

* Expressed as mean years \pm standard deviation.

${ }^{\dagger}$ Patients could choose more than one answer.

only two aborigines. In total, around $80 \%$ of the patients admitted having religious beliefs, mostly in Buddhism; and about $65 \%$ had an educational level up to junior high school. Most patients were married $(71.9 \%)$.

Economic characteristics of these patients including vocational status, monthly income, and sources of financial support are shown in Table 2. In Taiwan, people usually retire at the age of 65 years, and the mean monthly income is around 41333 New Taiwan dollars (US \$1333). Of all the patients, about $46 \%$ were retired or jobless, and about $64 \%$ had no income; yet $37.6 \%$ could live by themselves without any financial support from others. Social characteristics of the patients are also shown in Table 2. When in difficulties, most patients could receive help from their family $(77.4 \%)$, followed by medical staff $(40.5 \%)$, and friends $(31.9 \%)$. Nearly all patients $(97.7 \%)$ could obtain social support from one source or another. More than half of the patients never or seldom joined any social activities $(60.4 \%)$.

Psychologically, most patients worried about their own health $(72.4 \%)$, followed by financial burden
TABLE 2. Economic and social characteristics of study HD patients

\begin{tabular}{|c|c|c|}
\hline & $\begin{array}{l}\text { Number of } \\
\text { patients }\end{array}$ & $\begin{array}{l}\text { Percentage } \\
(\%)\end{array}$ \\
\hline \multicolumn{3}{|l|}{ Work status } \\
\hline Vocationally inactive & 394 & 45.82 \\
\hline Vocationally active & 466 & 54.18 \\
\hline \multicolumn{3}{|l|}{ Monthly income } \\
\hline None & 533 & 63.76 \\
\hline$<$ NT $\$ 25000^{*}$ & 115 & 13.75 \\
\hline$\geqq \mathrm{NT} \$ 25000^{*}$ & 188 & 22.49 \\
\hline \multicolumn{3}{|l|}{ Sources of financial support ${ }^{\dagger}$} \\
\hline Oneself & 324 & 37.63 \\
\hline Husband/wife & 243 & 28.22 \\
\hline Relatives (including children) & 252 & 29.27 \\
\hline Government & 160 & 18.58 \\
\hline Others & 37 & 4.30 \\
\hline \multicolumn{3}{|l|}{ Sources of social support ${ }^{\dagger}$} \\
\hline Family & 666 & 77.35 \\
\hline Medical staff & 349 & 40.53 \\
\hline Friends & 275 & 31.94 \\
\hline Religion & 2 & 0.23 \\
\hline Teachers & 1 & 0.12 \\
\hline \multicolumn{3}{|l|}{ Number of social support } \\
\hline 0 & 20 & 2.32 \\
\hline 1 & 457 & 53.08 \\
\hline 2 & 250 & 29.04 \\
\hline$\geqq 3$ & 134 & 15.56 \\
\hline \multicolumn{3}{|l|}{ Frequency of joining social activities } \\
\hline Never/seldom & 508 & 60.40 \\
\hline Occasionally/frequently & 333 & 39.60 \\
\hline
\end{tabular}

* NT, New Taiwan dollars.

${ }^{\dagger}$ Patients could choose more than one answer.

(18.8\%), and family (17.7\%), as shown in Table 3. Only 70 patients $(8.1 \%)$ did not worry about anything and none worried about social security as it is generally good in Taiwan. The mean score of the 352 patients

TABLE 3. Psychological characteristics of study HD patients

\begin{tabular}{lcc}
\hline & $\begin{array}{c}\text { Number of } \\
\text { patients }\end{array}$ & $\begin{array}{c}\text { Percentage } \\
(\%)\end{array}$ \\
\hline Worries* & & \\
Health & 623 & 72.36 \\
Economic burden & 162 & 18.82 \\
Family & 152 & 17.65 \\
Job & 54 & 6.27 \\
Marriage & 27 & 3.14 \\
Others & 7 & 0.08 \\
Number of worries & 70 & 8.13 \\
0 & 567 & 65.86 \\
1 & 168 & 19.51 \\
2 & 56 & 6.50 \\
$\geqq 3$ & & \\
Severity of depression & 139 & 39.49 \\
No depression (BDI $<14)$ & 66 & 18.75 \\
Mild (BDI 14-19) & 73 & 20.74 \\
Moderate (BDI 20-28) & 74 & 21.02 \\
Severe (BDI $\geq 29)$ & & \\
\hline
\end{tabular}

* Patients could choose more than one answer. 
TABLE 4. Clinical characteristics of study HD patients

\begin{tabular}{|c|c|c|}
\hline & $\begin{array}{l}\text { Number of } \\
\text { patients }\end{array}$ & $\begin{array}{c}\text { Percentage } \\
(\%)\end{array}$ \\
\hline \multicolumn{3}{|l|}{ Causes of renal failure } \\
\hline Diabetes mellitus & 322 & 37.79 \\
\hline Chronic glomerulonephritis & 291 & 34.16 \\
\hline Hypertension & 92 & 10.80 \\
\hline Systemic lupus erythematosus & 15 & 1.76 \\
\hline Others & 132 & 15.49 \\
\hline \multicolumn{3}{|l|}{ Number of comorbidities } \\
\hline 0 & 426 & 49.48 \\
\hline 1 & 290 & 33.68 \\
\hline 2 & 107 & 12.43 \\
\hline$\geqq 3$ & 38 & 4.41 \\
\hline \multicolumn{3}{|l|}{ Number of symptoms } \\
\hline 0 & 76 & 8.91 \\
\hline 1 & 383 & 44.90 \\
\hline 2 & 236 & 27.67 \\
\hline$\geqq 3$ & 158 & 18.52 \\
\hline Laboratory data & Number of patients & Mean \pm SD \\
\hline Hematocrit (\%) & 849 & $29.75 \pm 4.19$ \\
\hline Iron $(\mu \mathrm{g} / \mathrm{dL})$ & 784 & $70.99 \pm 35.32$ \\
\hline TIBC $(\mu \mathrm{g} / \mathrm{dL})$ & 830 & $225.78 \pm 55.86$ \\
\hline Ferritin (ng/mL) & 823 & $529.68 \pm 528.05$ \\
\hline Albumin (g/dL) & 843 & $4.06 \pm 0.39$ \\
\hline GOT (U/L) & 800 & $21.10 \pm 14.44$ \\
\hline GPT (U/L) & 835 & $20.57 \pm 23.43$ \\
\hline Phosphorus (mg/dL) & 851 & $5.01 \pm 1.51$ \\
\hline Uric acid (mg/dL) & 814 & $7.59 \pm 1.51$ \\
\hline iPTH (ng/L) & 767 & $185.80 \pm 239.55$ \\
\hline Triglyceride (mg/dL) & 838 & $169.42 \pm 130.40$ \\
\hline Cholesterol (mg/dL) & 836 & $188.30 \pm 46.34$ \\
\hline $\mathrm{Kt} / \mathrm{V}_{\text {urea }}$ & 794 & $1.41 \pm 0.29$ \\
\hline
\end{tabular}

GOT, aspartate aminotransferase; GPT, alanine aminotransferase; iPTH, intact parathyroid hormone; TIBC, total iron-binding capacity.

who completed the BDI was $19.2 \pm 11.5$. Depression was detected in around $60.5 \%$ of these patients.

Table 4 shows the clinical characteristics of all patients. The mean body height was $160.0 \pm 8.2 \mathrm{~cm}$ and the mean body weight was $56.3 \pm 10.3 \mathrm{~kg}$. Diabetes mellitus and chronic glomerulonephritis were the main causes of renal failure. Hypertension, angina, myocardial infarction, congestive heart failure, and peripheral arterial occlusive disease were common cardiovascular comorbidities (45.3\%). Mean duration on dialysis therapy was $6.0 \pm 4.3$ years, and $91.1 \%$ of the patients received erythropoietin therapy for their renal anemia. Around $91 \%$ of all patients reported symptoms such as pruritus $(36.8 \%)$, insomnia $(31.0 \%)$, and fatigue (30.6\%). From the laboratory data as in Table 4, the mean serum albumin level was $4.1 \pm 0.4 \mathrm{~g} / \mathrm{dL}$ and the mean $\mathrm{Kt} / \mathrm{V}_{\text {urea }}$ was $1.4 \pm 0.3$.

Mean values of the eight dimensions of SF-36 from all patients are displayed in Table 5. Scores from the general population in Taiwan are shown for comparison.

In the univariable linear regression, many socioeconomic factors were significantly associated with most of the SF-36 dimensions. However, after adjusting for clinical covariates and other significant predictors, only the following were found significantly associated with HRQOL (Table 6): older age was significantly inversely associated with $\mathrm{PF}(P<0.01)$, RP $(P<0.05)$, VT $(P<0.01)$, and SF $(P<0.05)$; higher monthly income (NT\$<25 000 vs. none) was significantly positively associated with $\operatorname{RE}(P<0.05)$ and MH $(P<0.05)$; increased frequency of joining social activities was significantly positively associated with

TABLE 5. Mean values of the dimensions of SF-36 of study HD patients and the general Taiwan population

\begin{tabular}{|c|c|c|}
\hline \multirow[b]{2}{*}{ Variable } & Scores of HD patients & Scores of population* \\
\hline & mean $\pm \mathrm{SD}$ & mean $\pm \mathrm{SD}$ \\
\hline $\mathrm{PF}$ & $47.90 \pm 30.49$ & $92.24 \pm 16.16$ \\
\hline RP & $37.57 \pm 44.41$ & $83.65 \pm 33.27$ \\
\hline $\mathrm{BP}$ & $67.50 \pm 27.49$ & $84.84 \pm 19.42$ \\
\hline $\mathrm{GH}$ & $43.10 \pm 24.07$ & $69.29 \pm 21.27$ \\
\hline VT & $48.32 \pm 22.43$ & $68.27 \pm 18.66$ \\
\hline $\mathrm{SF}$ & $60.96 \pm 27.26$ & $86.81 \pm 17.05$ \\
\hline $\mathrm{RE}$ & $49.20 \pm 46.16$ & $79.40 \pm 36.07$ \\
\hline $\mathrm{MH}$ & $59.04 \pm 21.46$ & $73.01 \pm 16.55$ \\
\hline
\end{tabular}

* Adapted from Tseng et al. 2003 (11). 


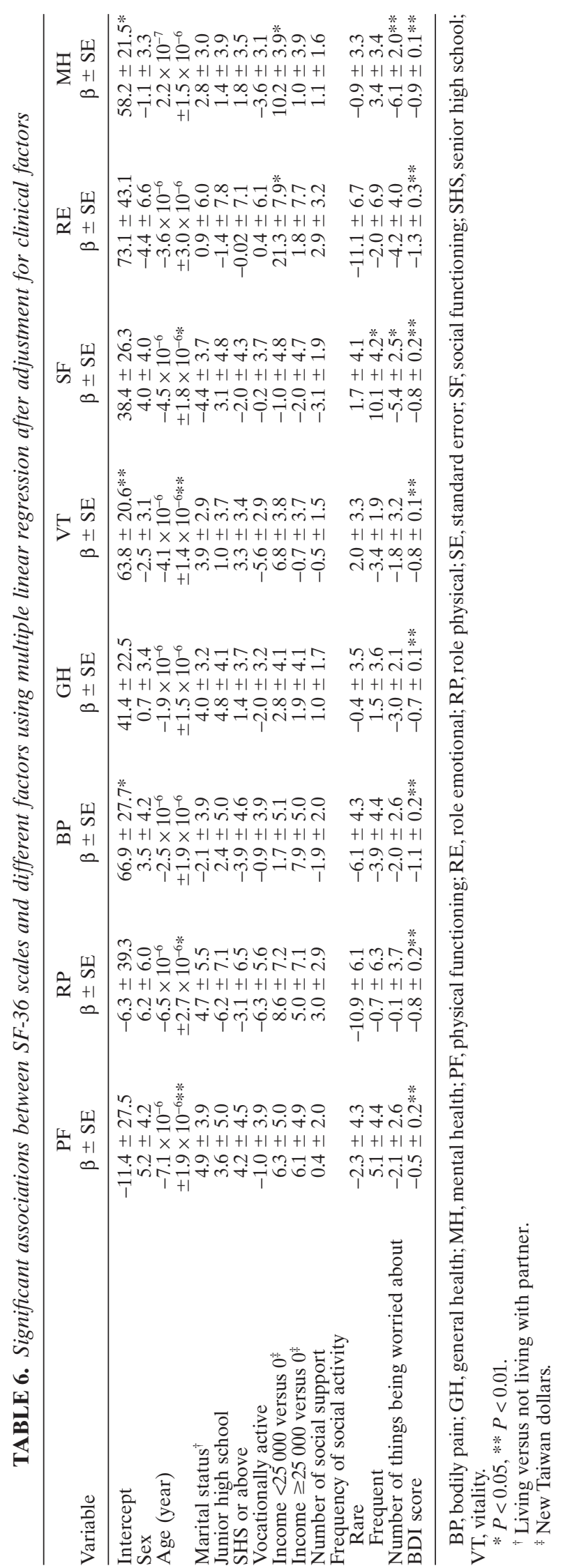

SF $\quad(P<0.05)$; more worries was significantly inversely associated with $\mathrm{SF}(P<0.05)$ and $\mathrm{MH}$ $(P<0.01)$; and a higher BDI score was significantly inversely associated with all dimensions of the SF-36 $(P<0.01)$.

\section{DISCUSSION}

Compared with the general population in Taiwan, HRQOL of the HD patients in this study was impaired for all SF-36 dimensions (Table 5). Molsted et al. demonstrated similar results in their local study (3).

Sex difference was found insignificant in the multivariable regression analyses of this study. Similar to previous cross-sectional (16) and prospective follow-up (17) studies, the result suggested that HRQOL scores were independent of gender.

The multivariable linear regression of this study showed that older age was significantly inversely associated with PF, RP, VT, and SF, but not MH. Previous studies conducted in different countries also demonstrated that age was strongly inversely associated with the physical domain scores (18). As age increases in the elderly, physical function of the body decreases (11). This applies to our study HD patients as well and thus the significant inverse association between age and domains reflecting physical function (PF, RP, and VT). Limitations of work and social activities also become more prominent among aged people. Rehabilitation programs, which aim to restore patients' work capacity and social function, can alleviate the impact of ageing on both RP and SF QOL. As age is an unmodifiable risk factor for worse HRQOL, older HD patients, whose muscle mass has decreased and vitality lessened, should have more attention and encouragement about keeping physically fit from medical staff in order that part of their VT QOL scores may be preserved.

Marital status was not associated with HRQOL of HD patients in this study. In a study on Hong Kong dialysis patients using the Chinese Dialysis QOL Scale, marital status also appeared to have no relation to QOL (19). As our study found the numbers of both single $(10.5 \%)$ and divorced $(4.3 \%)$ patients were relatively low in comparison with married patients $(71.9 \%)$, and the times of divorce or remarrying were not recorded, further investigations may be needed to clarify the causal relationship between marital status and HRQOL.

In this study, educational level was not associated with HRQOL in the multivariable regression analyses despite previous studies from which higher educational level was reported as significantly associated 
with better HRQOL $(4,20)$. Further study is needed to clarify their causal relationship.

Work status in this study was found insignificantly associated with HRQOL using multivariable regression analyses, in contrast to previous studies that demonstrated that vocationally active ESRD patients had higher QOL $(20,21)$. The discrepancy may be due to the fact that HD patients who can work are generally younger and have better physical function, that is, healthy worker effect may be present. Moreover, when compared with other stronger correlators of HRQOL such as age and depression, nonworking status has a relatively small effect and appeared only in some QOL scales (21).

Through the multivariable regression analyses, this study showed that higher monthly income was significantly positively associated with RE and MH scores. Moreno et al., in their multicenter cross-sectional study, and Sesso et al., in their prospective cohort study, also found that higher socioeconomic level was significantly related to better QOL $(20,22)$. ESRD patients in many countries have to pay for all or most of the dialysis treatment costs themselves, whereas HD patients in Taiwan enjoy free yet high-quality dialysis under current government policy. Financial burden was therefore less prominent in HD patients of this study when compared with other factors associated with HRQOL. Additionally, strong family support as reported in this study can explain why physical-related QOL dimensions were less affected by low monthly income.

In the multivariable regression analyses of this study, social support was not significantly associated with HRQOL. Previous studies suggested that better social support was correlated with higher HRQOL scores $(7,21)$, whereas a study by Kimmel et al. showed that responses to the Support Network Scale correlated positively only with scores on some of the McGill QOL subscales, but not the Satisfaction With Life Scale (23). The differences might be because of two reasons: first, the methods for measuring the strength of social support were inadequate, for example, Kimmel's Support Network Scale was not well validated whereas this study counted solely the number of sources of social support and assumed that more sources represented stronger support; and second, patients of this study had good social support generally (about $97 \%$ had at least one source of social support), that is, the difference in HRQOL scores between patients with and without social support was not distinct and could not be demonstrated clearly.

Increased frequency of joining social activities was found positively associated with the SF scores $(P<$ $0.05)$ in this study. Similarly, Wolcott et al. demon- strated in their study that a higher rate of participation in community activities was significantly correlated with higher QOL (6). In fact, physically as well as mentally healthier HD patients are willing to join social activities, especially outdoor ones that require more physical strength. As reluctance to join any social activity may be an early sign of physical function deterioration and/or depression, HD patients should be encouraged to take part in these activities to enhance their HRQOL.

Using the multivariable regression analyses, a greater number of worries was significantly inversely associated with SF $(P<0.05)$ and $\mathrm{MH}(P<0.01)$ scores. Worries can lead to decreased appetite, poor sleep, depressive mood, reduced concentration, and decreased interest in surrounding people and things. A decreased scoring of SF and MH may be the result. On the other hand, increased limitation of social functioning may be a source of worries itself. As most HD patients of this study worried about their own physical health, economic status, and family, it can be easily understood why they had significantly lower scores of MH. Psychological consultation, psychotherapy, and better mental support should be given to HD patients to improve their MH QOL.

BDI scores were significantly and strongly inversely associated with all the eight dimensions of SF-36 in both univariable and multivariable regression analyses $(P<0.01)$. It means that depression is the most important factor that is associated with HRQOL of our HD patients. This result confirmed Vázquez's report that concluded that psychological factors including depressive symptoms are crucial HRQOL determinants (21). Currently, depression is believed to be the most common psychiatric problem in HD patients (24). Around $60.5 \%$ of the patients in this study who had completed the BDI suffered from depression of various degrees. This prevalence rate was much higher than that reported by Taskapan of Turkey (35\%) (25). Depression can have adverse effects on HD patients' nutrition, immunology, dialysis compliance, and social interaction. Poor physical and emotional status will certainly further aggravate depression. As depression affects dialysis outcome and survival (23), early detection and proper management of depression should be emphasized and practiced.

This study was the first in Taiwan that successfully evaluated the associations between economic, social, psychological factors, and HRQOL of HD patients using the SF-36. Its sample size was large enough to truly reflect the HRQOL status of HD patients in Taiwan. To our best knowledge, it was also the first study to find that having "more worries" is signifi- 
cantly inversely associated with the mental HRQOL of HD patients. Medical staff should pay more attention to elder HD patients who have worse HRQOL. Regular assessment of HD patients' psychological condition, especially the severity of depression, should also be carried out at every dialysis center. Early and appropriate treatment of depression will hopefully improve the HRQOL as well as survival of HD patients in Taiwan.

A few limitations were noted in this study. First of all, like many other studies investigating HRQOL in ESRD, this study was cross-sectional and could not differentiate the exact causal relationships between various factors and HRQOL. Second, as patients who were too sick-of whom the HRQOL scores were expected to be lower-were excluded, valuable information about this subgroup might have been missed. Third, other validated objective questionnaires were not used for grading variables such as socioeconomic status and social support as reliable questionnaires of the kind are still lacking.

\section{CONCLUSIONS}

HRQOL of HD patients in northern Taiwan is significantly associated with socioeconomic condition and psychological status. After adjustments for variables of interest, higher monthly income, and joining more social activities are significantly positively associated with HRQOL, whereas more worries and deeper depression are significantly inversely associated with HRQOL.

Acknowledgments: We would like to thank the Ta-Tung Kidney Research Fund as well as Mrs. HsiuChin Lee Kidney Research Fund for supporting this investigation. We would also like to express our gratitude to all the physicians and nurses from different study hospitals for collecting the patients' data. The $14 \mathrm{HD}$ centers that joined the study were Cathay General Hospital, Da Chien General Hospital, En Chu Kong Hospital, Far Eastern Memorial Hospital, Far Eastern Poly-Clinic, Miao-Li General Hospital, National Taiwan University Hospital, Shin Kong Wu Ho-Su Memorial Hospital, Taipei County Hospital, Taipei Medical University Hospital, Taipei Municipal Hoping Hospital, Taipei Municipal Jen-Ai Hospital, Taipei Municipal Zhongxiao Hospital, and Yan Chai Hospital.

\section{REFERENCES}

1. Lopes AA, Bragg-Gresham JL, Satayathum S, et al. Worldwide Dialysis Outcomes and Practice Patterns Study Committee. Health-related quality of life and associated out- comes among hemodialysis patients of different ethnicities in the United States: the Dialysis Outcomes and Practice Patterns Study (DOPPS). Am J Kidney Dis 2003;41:605-15.

2. Tell GS, Mittelmark MB, Hylander B, Shumaker SA, Russell G, Burkart JM. Social support and health-related quality of life in black and white dialysis patients. ANNA J 1995;22: $301-8$.

3. Molsted S, Aadahl M, Schou L, Eidemak I. Self-rated health and employment status in chronic hemodialysis patients. Scand J Urol Nephrol 2004;38:174-8.

4. Manns B, Johnson JA, Taub K, Mortis G, Ghali WA, Donaldson C. Quality of life in patients treated with hemodialysis or peritoneal dialysis: what are the important determinants? Clin Nephrol 2003;60:341-51.

5. Valderrabano F, Jofre R, Lopez-Gomez JM. Quality of life in end-stage renal disease patients. Am J Kidney Dis 2001;38: 443-64.

6. Wolcott DL, Nissenson AR, Landsverk J. Quality of life in chronic dialysis patients. Factors unrelated to dialysis modality. Gen Hosp Psychiatry 1988;10:267-77.

7. Tovbin D, Gidron Y, Jean T, Granovsky R, Schnieder A. Relative importance and interrelations between psychosocial factors and individualized quality of life of hemodialysis patients. Qual Life Res 2003;12:709-17.

8. Ware JE Jr, Sherbourne CD. The MOS 36-item Short Form Health Survey (SF-36): I. Conceptual frame-work and item selection. Med Care 1992;30:473-83.

9. Lowrie EG, Curtin RB, LePain N, Schatell D. Medical outcomes study short form-36: a consistent and powerful predictor of morbidity and mortality in dialysis patients. Am J Kidney Dis 2003;41:1286-92.

10. Yu J, Coons SJ, Draugalis JR, Ren XS, Hays RD. Equivalence of Chinese and US-English versions of the SF-36 Health Survey. Qual Life Res 2003;12:449-57.

11. Tseng HM, Lu JFR, Tsai YJ. Assessment of health-related quality of life in Taiwan (II): norming and validation of SF-36 Taiwan version. Taiwan J Public Health 2003;22:512-8.

12. Chen YC, Hung KY, Kao TW, Tsai TJ, Chen WY. Relationship between dialysis adequacy and quality of life in long-term peritoneal dialysis patients. Perit Dial Int 2000;20:534-40.

13. Kao TW, Tsai DM, Wu KD, Shiah CJ, Hsieh BS, Chen WY. Impact of religious activity on depression and quality of life of chronic peritoneal dialysis patients in Taiwan. J Formos Med Assoc 2003;102:127-30.

14. Beck AT, Steer RA, Garbin MG. Psychometric properties of the Beck Depression Inventory: twenty-five years of evaluation. Clin Psychol Rev 1988;8:77-100.

15. Finkelstein FO, Finkelstein SH. Depression in chronic dialysis patients: assessment and treatment. Nephrol Dial Transplant 2000;15:1911-3.

16. Wasserfallen JB, Halabi G, Saudan P, et al. Quality of life on chronic dialysis: comparison between hemodialysis and peritoneal dialysis. Nephrol Dial Transplant 2004;19:1594-9.

17. de Jonge P, Ruinemans GM, Huyse FJ, ter Wee PM. A simple risk score predicts poor quality of life and non-survival at 1 year follow-up in dialysis patients. Nephrol Dial Transplant 2003;18:2622-8.

18. Blake C, Codd MB, Cassidy A, O'Meara YM. Physical function, employment and quality of life in end-stage renal disease. J Nephrol 2000;13:142-9.

19. Suet-Ching WL. The quality of life for Hong Kong dialysis patients. J Adv Nurs 2001;35:218-27.

20. Moreno F, Lopez Gomez JM, Sanz-Guajardo D, Jofre R, Valderrabano F. Quality of life in dialysis patients: a Spanish multicenter study. Spanish Cooperative Renal Patients Quality of Life Study Group. Nephrol Dial Transplant 1996;11(Suppl. 2):S125-129.

21. Vázquez I, Valderrábano F, Jofré R, et al. Spanish Cooperative Renal Patients Quality of Life Study Group. Psychosocial factors and quality of life in young hemodialysis patients with low comorbidity. J Nephrol 2003;16:886-94. 
22. Sesso R, Rodrigues-Neto JF, Ferraz MB. Impact of socioeconomic status on the quality of life of ESRD patients. Am J Kidney Dis 2003;41:186-95.

23. Kimmel PL, Peterson RA, Weihs KL, et al. Multiple measurements of depression predict mortality in a longitudinal study of chronic hemodialysis patients. Kidney Int 2000;57:2093-8.
24. Janssen van Doorn K, Heylen M, Mets T, Verbeelen D. Evaluation of functional and mental state and quality of life in chronic hemodialysis patients. Int Urol Nephrol 2004;36:263-7.

25. Taskapan H, Ates F, Kaya B, et al. Psychiatric disorders and large interdialytic weight gain in patients on chronic hemodialysis. Nephrology (Carlton) 2005;10:15-20. 